\title{
Studying the low-energy excess in cosmic-ray iron: a possible evidence of a massive supernova activity in the solar neighborhood via primary ${ }^{60} \mathrm{Fe}$
}

\author{
Nicolo Masi, ${ }^{a, b, *}$ M. J. Boschini, ${ }^{c, d}$ S. Della Torre, ${ }^{c}$ M. Gervasi, ${ }^{c, e}$ D. Grandi, ${ }^{c, e}$ \\ G. Jóhannesson, ${ }^{f, g}$ G. La Vacca, ${ }^{c, e}$ I. V. Moskalenko, ${ }^{h, i}$ S. Pensotti, ${ }^{c, e}$ \\ T. A. Porter, ${ }^{h, i}$ L. Quadrani, ${ }^{a, b}$ P. G. Rancoita, ${ }^{c}$ D. Rozza ${ }^{c, e}$ and M. Tacconic ${ }^{c, e}$ \\ ${ }^{a}$ Physics Department, University of Bologna, Via Irnerio, 46, Bologna, Italy \\ ${ }^{b}$ INFN, Bologna, Italy \\ ${ }^{c}$ INFN, Milano-Bicocca, Milano, Italy \\ ${ }^{d}$ CINECA, Segrate, Milano, Italy \\ ${ }^{e}$ Physics Department, University of Milano-Bicocca, Milano, Italy \\ ${ }^{f}$ Science Institute, University of Iceland, Dunhaga 3, IS-107 Reykjavik, Iceland \\ ${ }^{g}$ NORDITA, Roslagstullsbacken 23, 10691 Stockholm, Sweden \\ ${ }^{h}$ Hansen Experimental Physics Laboratory, Stanford University, Stanford, CA 94305 \\ ${ }^{i}$ Kavli Institute for Particle Astrophysics and Cosmology, Stanford University, Stanford, CA 94305 \\ E-mail: masinabo.infn.it
}

Since its launch the Alpha Magnetic Spectrometer-02 (AMS-02) has delivered outstanding quality measurements of the spectra of cosmic-ray (CR) species, which resulted in a number of breakthroughs. A recently published spectrum of iron is of a particular interest. Because of the large fragmentation cross section and large ionization energy losses, most of CR iron at low energies is local, and may harbor some features associated with relatively recent supernova (SN) activity inside the Local Bubble. Indeed, our analysis of AMS-02 measurements together with Voyager 1 and ACE-CRIS data reveals an unexpected bump in the iron spectrum and in the $\mathrm{Fe} / \mathrm{He}, \mathrm{Fe} / \mathrm{O}$, and $\mathrm{Fe} / \mathrm{Si}$ ratios at 1-2 GV. This is the first time when the excess is found in the elemental CR iron that is dominated by a stable ${ }^{56} \mathrm{Fe}$ isotope. Previously discovered were only deposits of radioactive ${ }^{60} \mathrm{Fe}$ in terrestrial and lunar samples, and in CRs. To confirm the excess and clarify its nature, precise measurements of other heavy CR species are of crucial importance. In this paper, we employ the GalProp-HelMod framework to derive the ${ }^{60} \mathrm{Fe} /{ }^{56} \mathrm{Fe}$ ratio in the sources using ${ }^{60} \mathrm{Fe}$ abundance from ACE-CRIS and compare it with the SN yield. We also provide an updated local interstellar spectrum (LIS) of iron in the energy range from $1 \mathrm{MeV}$ nucleon $^{-1}$ to $\sim 10 \mathrm{TeV}_{\text {nucleon }}^{-1}$ and the sub-Fe/Fe ratio calculated in the same framework.

$37^{\text {th }}$ International Cosmic Ray Conference (ICRC 2021)

July 12th-23rd, 2021

Online - Berlin, Germany

\footnotetext{
${ }^{*}$ Presenter
} 


\section{Introduction}

New era of precise astrophysical measurements has started about a decade ago with the launch of the Payload for Antimatter Matter Exploration and Light-nuclei Astrophysics (PAMELA). It was followed by a continuing series of launches of unique instrumentation, such as the Fermi Large Area Telescope (Fermi-LAT), AMS-02, NUCLEON experiment, CALorimetric Electron Telescope (CALET), DArk Matter Particle Explorer mission (DAMPE), and Cosmic-Ray Energetics and Mass investigation (ISS-CREAM). These experiments are operating in the high-energy and very-highenergy domains. Meanwhile, understanding the origin of cosmic rays (CRs) and our interstellar environment is impossible without connecting high energy measurements with data from lowenergy experiments, such as the Cosmic Ray Isotope Spectrometer onboard of the Advanced Composition Explorer (ACE-CRIS) operating at the L1 Lagrange point for more than two decades, and Voyager 1, 2 spacecraft, which are now in the interstellar space.

The precisely measured spectra of light nuclei through Si by AMS-02 confirmed the earlier discoveries of breaks in the $p$ and He spectra [1, 17, 47], and revealed new features, such as the grouping of spectral indices by the origin of elements (primary, secondary, and intermediate group) $[3,4,5,6,7,8,9,10,11,12,13]$. Particularly interesting is the origin of a small but significant difference in indices of oxygen $(\mathrm{C}, \mathrm{O})$ and silicon $(\mathrm{Ne}, \mathrm{Mg}, \mathrm{Si})$ groups of mainly primary species.

One of the latest long awaited surprises from the series of new experiments is the spectrum of iron published by AMS-02 [14]. Because of the large fragmentation cross section of iron and large ionization energy losses at low energies, most of low-energy CR iron is local, coming from relatively recent supernova (SN) activity in the solar neighborhood. The injection of iron is quite different from the injection of lighter species-it is injected into the interstellar space at the time of a SN explosion, while lighter elements are abundant in pre-supernova winds of massive stars. Besides, it has lower charge to mass ratio $(Z / A)_{\mathrm{Fe}} \approx 0.46$ compared to $(Z / A)_{\mathrm{He}, \mathrm{C}, \mathrm{O}, \mathrm{Si}} \approx 1 / 2$ for lighter species and $(Z / A)_{p}=1$ for protons. Altogether it may result in the $\mathrm{SN}$ shock acceleration of iron to be somewhat different from other nuclei. It is, therefore, natural to expect that the spectrum of CR iron could be different from other species, and we emphasized that numerous times [25].

In a recent paper [25] we put forward a set of predicted spectra for all CR nuclei $\mathrm{H}-\mathrm{Ni}$, including those that are not yet published by AMS-02. Our predicted spectra for those species were based on Voyager 1 [28] and ACE-CRIS data at low energies, while at high energies we used ATIC-2 [47], CREAM [15], NUCLEON [38, 39], CALET [2], and DAMPE [18]. In the intermediate range we used the a subset of HEAO-3-C2 data [30] that correspond to the aerogel counter of HEAO3-C2 experiment. Our comparison with the published AMS-02 spectra of $\mathrm{H}-\mathrm{O}, \mathrm{Ne}, \mathrm{Mg}, \mathrm{Si}$ has shown that the HEAO-3-C2 data in the middle range from $2.65-10.6 \mathrm{GeV}_{\text {nucleon }}{ }^{-1}$, the so-called "plateau" corresponding to the aerogel counter, agree with the AMS-02 data quite well while at lower and higher energies the systematic deviations are large [25]. However, the newly measured precise spectrum of iron just published by AMS-02 [14] is indeed harboring a surprise that provides an exciting opportunity to gain insight into the origin and evolution of the local interstellar medium.

In this paper, we employ the GALPROP ${ }^{1}-\mathrm{HeLMoD}^{2}$ framework that is proved to be a reliable tool in deriving the LIS of CR species [21, 25].

${ }^{1}$ Available from http://galprop.stanford.edu

${ }^{2} \mathrm{http} / / /$ www.helmod.org/ 


\section{Calculations}

Here we are using the same CR propagation model with distributed reacceleration and convection that was used in our previous analyses $[20,22,23,24,25]$. The values of propagation parameters along with
Table 1 Best-fit propagation parameters for the $I$-scenario

\begin{tabular}{rlcc}
\hline \hline Parameter & Units & Best Value & Error \\
\hline$z_{h}$ & $\mathrm{kpc}$ & 4.0 & 0.6 \\
$D_{0}(R=4 \mathrm{GV})$ & $\mathrm{cm}^{2} \mathrm{~s}^{-1}$ & $4.3 \times 10^{28}$ & 0.7 \\
$\delta$ & & 0.415 & 0.025 \\
$V_{\text {Alf }}$ & $\mathrm{km} \mathrm{s}^{-1}$ & 30 & 3 \\
$d V_{\text {conv }} / d z$ & $\mathrm{~km} \mathrm{~s}^{-1} \mathrm{kpc}^{-1}$ & 9.8 & 0.8 \\
\hline
\end{tabular}

their confidence limits are derived from the best available CR data using the Markov Chain Monte Carlo (MCMC) routine. Five main propagation parameters, that affect the overall shape of CR spectra, were left free in the scan using GALPROP running in the 2D mode: the Galactic halo half-width $z_{h}$, the normalization of the diffusion coefficient $D_{0}$ at the reference rigidity $R=4 \mathrm{GV}$ and the index of its rigidity dependence $\delta$, the Alfvén velocity $V_{\text {Alf }}$, and the gradient of the convection velocity $d V_{\text {conv }} / d z\left(V_{\text {conv }}=0\right.$ in the plane, $\left.z=0\right)$. Their best-fit values tuned to the AMS-02 data are listed in Table 1 and are the same as obtained in Boschini et al. [25]. The radial size of the Galaxy does not significantly affect the values of propagation parameters and was set to $20 \mathrm{kpc}$. Besides, we introduced a factor $\beta^{\eta}$ in the diffusion coefficient, where $\beta=v / c$, and $\eta$ was left free. The best fit value of $\eta=0.70$ improves the agreement at low energies, and slightly affects the choice of injection indices $\gamma_{0}$ an $\gamma_{1}$. A detailed discussion of the injection $(I)$ and propagation ${ }^{3}(P)$ scenarios of the 350 GV break can be found in Vladimirov et al. [51] and Boschini et al. [25]. The corresponding B/C ratio also remains the same [25], and compares well with all available measurements: Voyager 1 [28], ACE-CRIS 4, AMS-02 [5], ATIC-2 [47], CREAM [15, 16], and NUCLEON [39].

In this calculation we are tuning only the injection spectrum of iron to match the new data from AMS-02 at high energies and ACE-CRIS and Voyager 1 at low energies. Compared to the previous injection spectrum derived from HEAO-3-C2 data [25] we have to add another break at low rigidities at $R^{\prime}=2.94 \mathrm{GV}$ and a very steep spectrum with index $\gamma^{\prime}=3.62$ between $R_{0}=2.00$ $\mathrm{GV}$ and $R^{\prime}=2.94 \mathrm{GV}$. The obtained agreement with data is good, but the new iron spectrum is quite different from what was expected based on the earlier fit that employs the HEAO-3-C2 data [25]. The primary abundance of ${ }^{56} \mathrm{Fe}$ is also increased from 515 to 577 [25], with relative abundances of isotopes of Fe after propagation tuned to ACE-CRIS data.

\section{Results}

Fig. 1 (left) shows the fit to the iron spectrum by AMS-02 [14] and the residuals. The right panel shows two fits as compared to the data sets by HEAO-3-C2 $2^{5}$ [30] or by AMS-02 [14] in the

${ }^{3}$ The $P$-scenario assumes a break in the diffusion coefficient with index $\delta_{1}=\delta$ (Table 1 ) below the break and index $\delta_{2}=0.15 \pm 0.03$ above the break at $R=370 \pm 25 \mathrm{GV}$ [20].

${ }^{4} \mathrm{http}: / /$ www.srl.caltech.edu/ACE/ASC/level2/cris_12desc.html

${ }^{5}$ While relying on the HEAO-3-C2 data [30] worked well for light and middle-range nuclei, it does not work so good for $\mathrm{Fe}-$ as we have learned after AMS-02 data were published [14]. Surprisingly all HEAO-3-C2 data points for Fe overlap with AMS-02 data (Fig. 1, right), but the solar modulation levels during the HEAO-3 flight and AMS-02 data taking are dramatically different. There are several possible reasons for such a discrepancy. The HEAO-3-C2 experiment was built in 1970s, at the same time that Voyager 1, 2 were built. There was no prelaunch detector response simulations, many systems went untested and the calibrations were done after the launch. In particular, the rigidity calibration was done using the geomagnetic field; the latter was lacking the detailed knowledge at that time [25]. While it is difficult to point to a single specific reason, we think that the incorrect evaluation of the probability of nuclear fragmentation is 

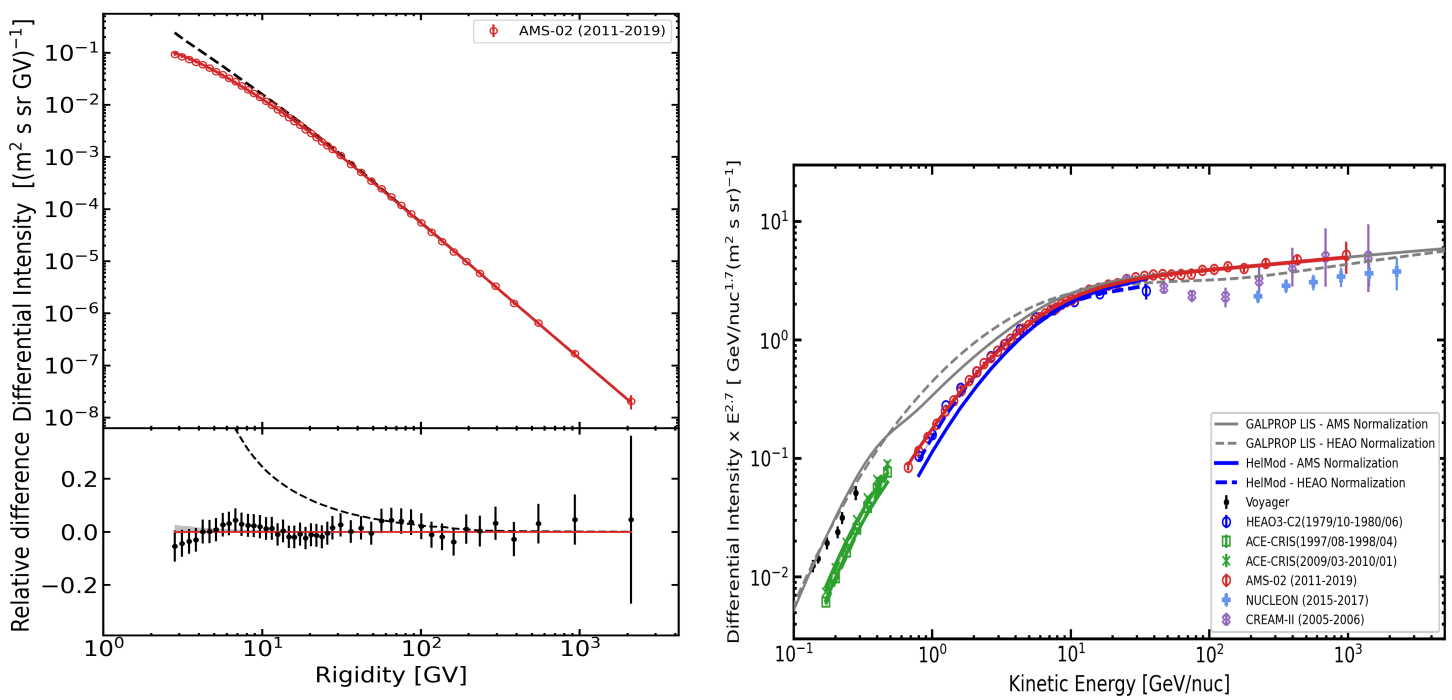

Figure 1 A fit to the AMS-02 spectrum of iron [14]. In the top left panel, only AMS-02 data are shown. The bottom left panel shows the quality of the fit, the relative difference between the calculations and the data set. The right panel shows two fits as compared to the data sets by HEAO3-C2 [30] or by AMS-02 [14] in the intermediate range, while at low and very-high energies we use Voyager 1 [28] / ACE-CRIS, and CREAM [15] / NUCLEON [38, 39] data, correspondingly. The gray lines show the LIS tuned to AMS-02 data (solid line) and the previous LIS tuned to the "plateau" middle range of the HEAO-3-C2 data (dashed line), while colored lines show the LIS modulated appropriately to the periods of ACE-CRIS (green), AMS-02 (red), and HEAO-3 (blue dashed) data taking. The solid blue line shows where the correct spectrum of HEAO-3-C2 should be if we assume the iron LIS based on AMS-02.

intermediate range, while at low and very-high energies we use Voyager 1 [28] / ACE-CRIS, and CREAM [15] / NUCLEON [38, 39] data, correspondingly. One can see that matching the Voyager 1 and modulated ACE-CRIS data, on one hand, and modulated AMS-02 data, on the other hand, requires a sharp spectral steepening in the transition region of the LIS between $\sim 2 \mathrm{GV}$ and $\sim 3 \mathrm{GV}$ and a similar feature in the injection spectrum as well.

A comparison of the $\mathrm{Fe} / \mathrm{He}, \mathrm{Fe} / \mathrm{O}$, and $\mathrm{Fe} / \mathrm{Si}$ ratios with AMS-02 data shows excellent agreement in the rigidity range from $2 \mathrm{GV}-2 \mathrm{TV}$ (Fig. 2). If the ratios are extended to lower energies where they compare with Voyager 1 and ACE-CRIS data, one can see a clear bump at $\sim 1-2$ GV. The bump appears in the energy gap between ACE-CRIS and AMS-02, and is the result of tuning the iron spectrum to all three data sets, Voyager 1, ACE-CRIS, AMS-02, each with an appropriate heliospheric modulation level. The anomaly would be unnoticed if the data of each instrument is

the most likely. Also the instrumental grammage is significant and the loss of heavy CR nuclei due to the fragmentation was considerable. Therefore, the accuracy of a charge changing cross section is crucial for heavy species while it is less essential for light nuclei. On the other hand, the AMS-02 experiment uses modern technology, its response was thoroughly simulated and tested, it also has several independent systems that allow for data cross checks. Therefore, it is rather unlikely to have a serious flaw. If our conclusion is correct, we should see a gradual increase in the discrepancy between the HEAO-3-C2 "plateau" data and AMS-02 data as the mass number increases. 

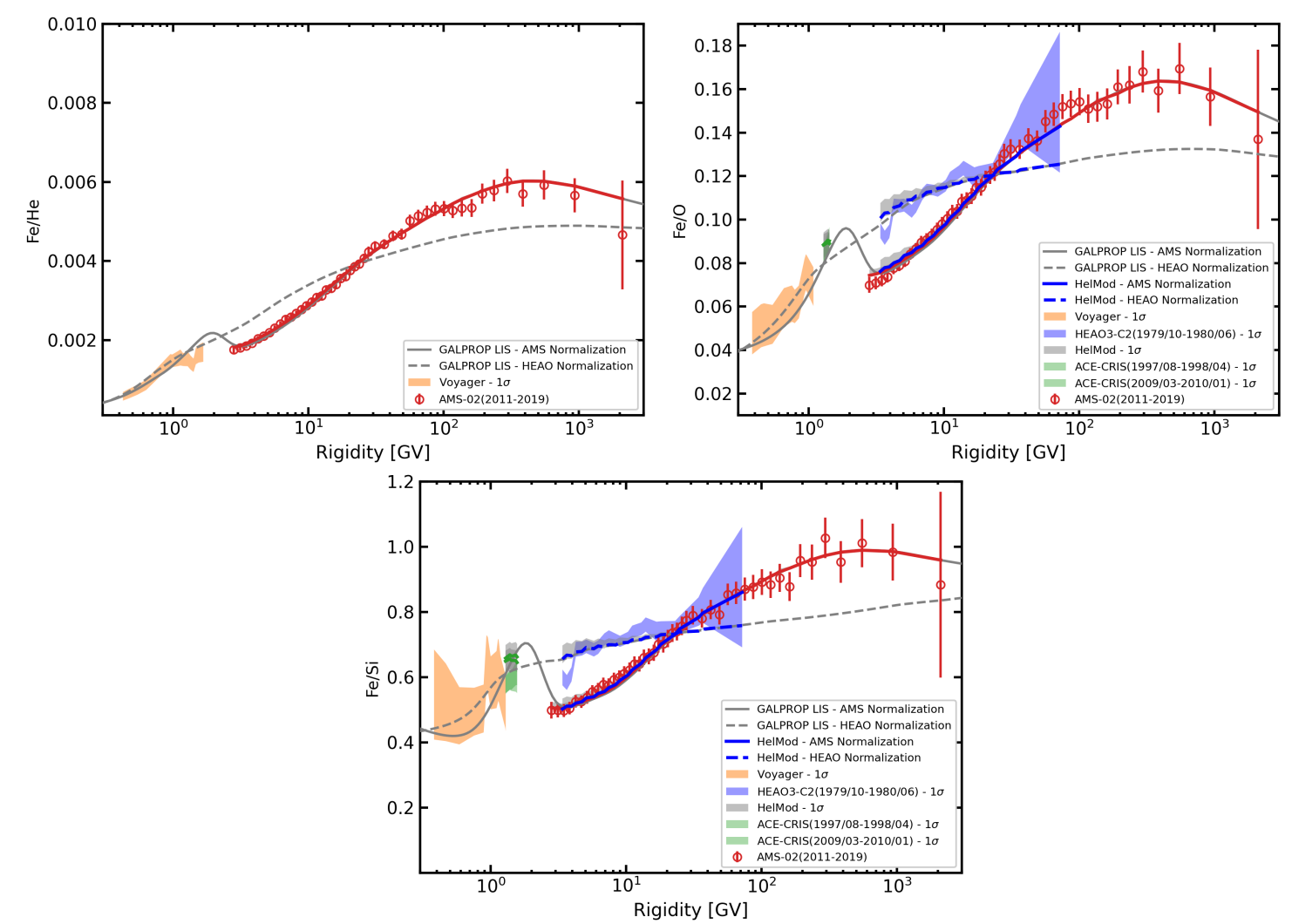

Figure 2 The calculated ratios of primary species, $\mathrm{Fe} / \mathrm{He}, \mathrm{Fe} / \mathrm{O}, \mathrm{Fe} / \mathrm{Si}$, are compared with Voyager 1 [28], ACE-CRIS, and AMS-02 data [12, 13, 14]. Also shown are HEAO-3-C2 data [30]. The gray line shows the LIS ratios tuned to AMS-02 data, and the red line shows the modulated LIS ratios. The dashed gray line shows the LIS ratios tuned to HEAO-3-C2 data [25] based on the "plateau" middle range of the HEAO-3-C2 data. For the Fe/He ratio, only Fe was tuned to the HEAO-3-C2 data. In all plots, the Voyager 1, ACE-CRIS, and HEAO-3-C2 data are converted from kinetic energy per nucleon to rigidity assuming $A / Z=2$. The shaded area shows the ratios modulated to the appropriate level (ACE-CRIS, HEAO-3-C2) with the width corresponding to $1 \sigma$ error.

taken separately.

The first ever accurate measurement of the iron spectrum in the rigidity range from $2 \mathrm{GV}-2 \mathrm{TV}$ [14] is a giant milestone. The appearance of the bump in the $\mathrm{Fe} / \mathrm{He}, \mathrm{Fe} / \mathrm{O}$, and $\mathrm{Fe} / \mathrm{Si}$ ratios implies a local source of low-energy CRs. Because of the fast ionization losses of iron nuclei in the ISM, $\mathrm{CR}$ iron from distant sources is suppressed and this gives the local source such a prominence.

The likely source of the excess CR iron are the old SN remnants. In fact, the evidence of the past $\mathrm{SN}$ activity in the local ISM is abundant. The Local Bubble is a low density region of the size of $\sim 200 \mathrm{pc}$ around the Sun filled with hot $\mathrm{H}$ i gas that itself was formed in a series of SN explosions $[29,35,45,48,50,54]$. There are multiple reports of an excess of radioactive ${ }^{60} \mathrm{Fe}$ found in the deep ocean sediments [31, 34, 41, 42, 44, 46, 52, 53], in lunar regolith samples [27, 32, 33], and more recently in the Antarctic snow [43]. Such deposits can be made by SN explosions in the solar neighborhood. Recent observation of ${ }^{60} \mathrm{Fe}$ [49] in CRs by ACE-CRIS spacecraft [19] implies that 

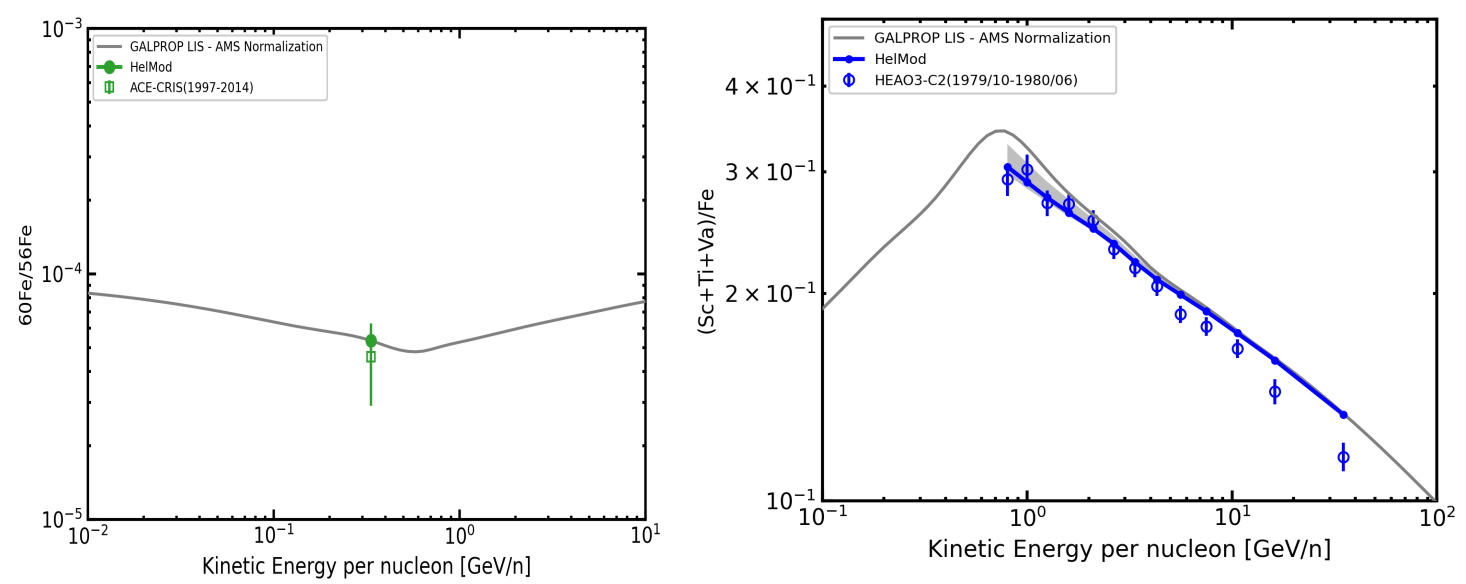

Figure 3 Left: The calculated ${ }^{60} \mathrm{Fe} /{ }^{56} \mathrm{Fe}$ ratio is compared with ACE-CRIS data. The gray solid line is tuned to the AMS-02 data. The green point shows the ratio modulated with HeLMod. Right: The calculated sub-Fe/Fe ratio, tuned to AMS-02 data, compared with HEAO-3-C2 data: the gray line is the LIS, and the blue line is the HeLMod modulated ratio.

the low-energy CRs from the most recent $\mathrm{SN}$ are still around. ACE-CRIS experiment measured a ${ }^{60} \mathrm{Fe} /{ }^{56} \mathrm{Fe}$ ratio of $(4.6 \pm 1.7) \times 10^{-5}$ near Earth and an inferred $(7.5 \pm 2.9) \times 10^{-5}$ at the CR source.

It is hard to establish the number of SNe events and their exact timing, but it is clear that there could be several events during the last $\sim 10 \mathrm{Myr}$ at distances of up to 100 parsecs [52]. The most recent SN events in the solar neighborhood were 1.5-3.2 Myr and 6.5-8.7 Myr ago [36, 52]. The measured spread (width) of the signal is $\sim 1.5 \mathrm{Myr}$ [36], which is too long for a single event. Each of these events could, in principle, consist of several consequent SN explosions separated by some 100 kyr, as an estimated time spread for a single SN, located at $\sim 100$ pc from the Earth, is just $\sim 100-400$ kyr and the travel time is $\sim 200 \mathrm{kyr}$. A detailed modeling by Breitschwerdt et al. [26] indicates two SNe at distances 90-100 pc with the closest occurred 2.3 Myr ago and the second-closest exploded about 1.5 Myr ago, both with stellar masses of $\sim 9 M_{\odot}$, while in [37] the authors infer from lunar ${ }^{60} \mathrm{Fe}$ deposition a progenitor SN occurring $2.8 \mathrm{Myr}$ ago within the Tuc-Hor stellar group, at a distance of $\sim 50 \mathrm{pc}$ and a mass of $8 \div 10 M_{\odot}$.

The new-found excess in the iron spectrum below $\sim 2 \mathrm{GV}$ is therefore falling in line with other excesses in ${ }^{60} \mathrm{Fe}$. However, this is the first time when the excess is found in the spectrum of the element that is dominated by stable species, most notably ${ }^{56} \mathrm{Fe}$. As illustrated in Fig. 3 left, the correct ACE-CRIS ${ }^{60} \mathrm{Fe} /{ }^{56} \mathrm{Fe}$ ratio could be reproduced within our model only assuming a primary ${ }^{60} \mathrm{Fe}$ component, with a normalized abundance at sources of about 0.05 w.r.t. 577 for ${ }^{56} \mathrm{Fe}$, i.e. ${ }^{60} \mathrm{Fe} /{ }^{56} \mathrm{Fe} \approx 8.7 \times 10^{-5}$. This is fully consistent with the aforementioned ACE-CRIS calculation.

The effective ${ }^{60} \mathrm{Fe} /{ }^{56} \mathrm{Fe}$ yield at the source represents an upper limit for computations, but it suffers at least one order of magnitude of uncertainty as a function of the ${ }^{59} \mathrm{Fe}(n, \gamma){ }^{60} \mathrm{Fe}$ cross section in the C-burning and He-burning shells of the star [40]. We believe that the iron excess we found in the AMS-02 data at $1-2 \mathrm{GV}$ is mainly due to ${ }^{56} \mathrm{Fe}$, although it would be trilling to measure the isotopic composition of iron at these rigidities. A likely scenario is that ${ }^{60} \mathrm{Fe}$ (half-life of $2.62 \mathrm{Myr}$ ) produced in a SN explosion was first dispersed in the ISM and accelerated by a shock 
wave from a subsequent SN [19], though a one-SN scenario cannot be excluded. The delay between the explosions cannot be much longer than the half-life of ${ }^{60} \mathrm{Fe}$ that has yet to propagate in the interstellar medium to reach the solar system. A detection of $\gamma$-ray emission lines associated with the decay of ${ }^{60} \mathrm{Fe}$ or ${ }^{60} \mathrm{Co}$, its $\beta^{-}$decay product, in the local Galaxy could provide some clues to the origin of the low-energy excesses of ${ }^{60} \mathrm{Fe}$ and atomic iron in CRs.

In Fig. 3 right, we show the calculated $(\mathrm{Sc}+\mathrm{Ti}+\mathrm{V}) / \mathrm{Fe}=$ sub-Fe/Fe ratio, where the iron spectrum is tuned to the AMS-02 data (Fig. 1), and the propagation parameters are summarized in Table 1. Because there are no AMS-02 measurements of sub-Fe species yet, we are have to use the HEAO3-C2 ratio that is only available in this range. The agreement with HEAO-3-C2 data below $10 \mathrm{GV}$ (the aerogel counter) is good thus corroborating the overall coherence of the "iron bump" scenario.

\section{Conclusion}

Using a combined data of AMS-02 [14], ACE-CRIS, and Voyager 1 [28], we found an unexpected bump in the spectrum of $\mathrm{CR}$ iron and the $\mathrm{Fe} / \mathrm{He}, \mathrm{Fe} / \mathrm{O}$, and $\mathrm{Fe} / \mathrm{Si}$ ratios, which is revealed as a sharp steepening in the range 2-3 GV necessary to connect ACE-CRIS and AMS-02 data. The new-found excess in the iron spectrum below $\sim 2 \mathrm{GV}$ is therefore falling in line with excesses in ${ }^{60} \mathrm{Fe}$ found in terrestrial and lunar samples, and in CRs. The found excesses are likely connected with the past SN activity in the Local Bubble. However, this is the first time when the excess is found in the spectrum of the element that is dominated by stable species. It is also important to measure the spectra of other heavy CR species, specifically in the low-middle 1-10 GV rigidity domain, to see if similar spectral features are present. To constrain the ${ }^{60} \mathrm{Fe}$ yield from $\mathrm{SNe}$ and to get a grip on the 2-3 Myr ago progenitor event, it would be useful to study a corresponding production of other long-lived radioactive isotopes, such as ${ }^{26} \mathrm{Al}$, and their ratios, e.g., ${ }^{60} \mathrm{Fe} /{ }^{26} \mathrm{Al}$ and ${ }^{26} \mathrm{Al} /{ }^{27} \mathrm{Al}$. Observation of the nuclear emission lines from the interstellar medium with a new generation of instrument would also be highly desirable.

\section{References}

[1] Adriani, O., Barbarino, G. C., Bazilevskaya, G. A., et al. 2011, Science, 332, 69

[2] Adriani, O., Akaike, Y., Asano, K., et al. 2019, PhRvL, 122, 181102

[3] Aguilar, M., \& et al. 2016, PhRvL, 117, 091103

[4] Aguilar, M., \& et al. 2016, PhRvL, 117, 231102

[5] Aguilar, M., \& et al. 2018, PhRvL, 120, 021101

[6] Aguilar, M., \& et al. 2018, PhRvL, 121, 051103

[7] Aguilar, M., \& et al. 2019, PhRvL, 122, 101101

[8] Aguilar, M., \& et al. 2019, PhRvL, 122, 041102

[9] Aguilar, M., Aisa, D., Alpat, B., et al. 2014, PhRvL, 113, 221102

[10] Aguilar, M., et al. 2015, PhRvL, 115

[11] Aguilar, M., et al. 2015, PhRvL, 114

[12] Aguilar, M., et al. 2017, PhRvL, 119, 251101

[13] Aguilar, M., et al. 2020, PhRvL, 124, 211102

[14] Aguilar, M., et al. 2021, PhRvL, 126, 041104

[15] Ahn, H. S., Allison, P. S., Bagliesi, M. G., et al. 2008, Astroparticle Physics, 30, 133

[16] Ahn, H. S., et al. 2009, ApJ, 707, 593

[17] Ahn, H. S., Allison, P., Bagliesi, M. G., et al. 2010, ApJ1, 714, L89

[18] An, Q., Asfandiyarov, R., Azzarello, P., et al. 2019, Science Advances, 5 
[19] Binns, W. R., Israel, M. H., Christian, E. R., et al. 2016, Science, 352, 677

[20] Boschini, M., et al. 2020, ApJ, 889, 167

[21] Boschini, M. J., Della Torre, S., Gervasi, M., La Vacca, G., \& Rancoita, P. G. 2019, Adv. Spa. Res., 64, 2459

[22] Boschini, M. J., \& et al. 2018, ApJ, 858, 61

[23] Boschini, M. J., \& et al. 2018, ApJ, 854, 94

[24] Boschini, M. J., et al. 2017, ApJ, 840, 115

[25] Boschini, M. J., Della Torre, S., Gervasi, M., et al. 2020, ApJS, 250, 27

[26] Breitschwerdt, D., Feige, J., Schulreich, M. M., et al. 2016, Nature, 532, 73

[27] Cook, D. L., Berger, E., Faestermann, T., et al. 2009, in Lunar and Planetary Science Conference, 1129

[28] Cummings, A. C., Stone, E. C., Heikkila, B. C., et al. 2016, ApJ, 831, 18

[29] Curtis, S., et al. 2018, The Astrophysical Journal, 870, 2

[30] Engelmann, J. J., Ferrando, P., Soutoul, A., et al. 1990, A\&A, 233, 96

[31] Feige, J., Wallner, A., Altmeyer, R., et al. 2018, Phys. Rev. Lett., 121, 221103

[32] Fimiani, L., et al. 2012, in Lunar and Planetary Science Conference, 1279

[33] Fimiani, L., et al. 2014, in Lunar and Planetary Science Conference, 1778

[34] Fitoussi, C., Raisbeck, G. M., Knie, K., et al. 2008, Phys. Rev. Lett., 101, 121101

[35] Frisch, P. C., Redfield, S., \& Slavin, J. D. 2011, ARA\&A, 49, 237

[36] Fry, B. J., Fields, B. D., \& Ellis, J. R. 2015, ApJ, 800, 71

[37] Fry, B. J., Fields, B. D., \& Ellis, J. R. 2016, The Astrophysical Journal, 827, 48

[38] Grebenyuk, V., Karmanov, D., Kovalev, I., et al. 2019, Advances in Space Research, 64, 2546

[39] Grebenyuk, V., et al. 2019, Advances in Space Research, 64, 2559

[40] Jones, S., et al. 2019, MNRAS, 485, 4287

[41] Knie, K., Korschinek, G., Faestermann, T., et al. 2004, PhRvL, 93, 171103

[42] Knie, K., et al. 1999, PhRvL, 83, 18

[43] Koll, D., Korschinek, G., Faestermann, T., et al. 2019, PhRvL, 123, 072701

[44] Koll, D., et al. 2020, EPJ Web Conf., 232, 02001

[45] Limongi, M., \& Chieffi, A. 2006, New Astronomy Reviews, 50, 474

[46] Ludwig, P., Bishop, S., Egli, R., et al. 2016, Proc. Nat. Acad. Sci., 113, 9232

[47] Panov, A. D., Adams, J. H., Ahn, H. S., et al. 2009, Bull. Russian Acad. Sci., Physics, 73, 564

[48] Prantzos, N. 2011, Nucleosynthesis and gamma-ray lines

[49] Rugel, G., Faestermann, T., Knie, K., et al. 2009, PhRvL, 103, 072502

[50] Sfeir, D. M., Lallement, R., Crifo, F., \& Welsh, B. Y. 1999, A\&A, 346, 785

[51] Vladimirov, A. E., Jóhannesson, G., Moskalenko, I. V., \& Porter, T. A. 2012, ApJ, 752, 68

[52] Wallner, A., Feige, J., Kinoshita, N., et al. 2016, Nature, 532, 69

[53] Wallner, A., Feige, J., Fifield, L. K., et al. 2020, Proc. Nat. Acad. Sci., 117, 21873

[54] Wang, X., Clark, A. M., Ellis, J., et al. 2021, r-Process Radioisotopes from Near-Earth Supernovae and Kilonovae, arXiv: 2105.05178 